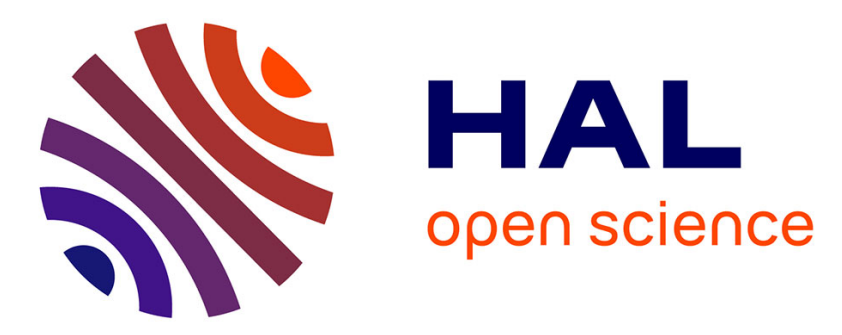

\title{
Context Awareness for Flexible Manufacturing Systems Using Cyber Physical Approaches
}

\author{
Sebastian Scholze, Jose Barata
}

\section{To cite this version:}

Sebastian Scholze, Jose Barata. Context Awareness for Flexible Manufacturing Systems Using Cyber Physical Approaches. 7th Doctoral Conference on Computing, Electrical and Industrial Systems (DoCEIS), Apr 2016, Costa de Caparica, Portugal. pp.107-115, 10.1007/978-3-319-31165-4_11 . hal01438234

\section{HAL Id: hal-01438234 \\ https://hal.inria.fr/hal-01438234}

Submitted on 17 Jan 2017

HAL is a multi-disciplinary open access archive for the deposit and dissemination of scientific research documents, whether they are published or not. The documents may come from teaching and research institutions in France or abroad, or from public or private research centers.
L'archive ouverte pluridisciplinaire HAL, est destinée au dépôt et à la diffusion de documents scientifiques de niveau recherche, publiés ou non, émanant des établissements d'enseignement et de recherche français ou étrangers, des laboratoires publics ou privés. 


\title{
Context Awareness for Flexible Manufacturing Systems Using Cyber Physical Approaches
}

\author{
Sebastian Scholze ${ }^{1}$, Jose Barata ${ }^{2}$ \\ ${ }^{1}$ Institut für angewandte Systemtechnik Bremen GmbH, 28359 Bremen, Germany \\ scholze@atb-bremen.de \\ ${ }^{2}$ Dep. Eng. Electrotecnica, Faculdade de Ciencias e Tecnologica, Universidade Nova de \\ Lisboa, 2829-516 Caparica, Portugal, jab@uninova.pt
}

\begin{abstract}
The work presented in this paper demonstrates how flexible manufacturing systems (FMS) combined with context awareness can be used to allow for an improved decision support in manufacturing industry. Thereby manufacturing companies shall be supported in a continuous process of increasing efficiency and availability of their production machines. Such optimization has to be embedded in the processes allowing for run time adaptation of the process to various dynamically changing external conditions. Context awareness, based on the information obtained from cyber physical systems, is a promising approach to allow for efficient building of such embedded optimization solutions. The objective of the research presented is to explore how context awareness, using the information from cyber physical systems integrated in the processes, can be applied to build a solution for selfoptimization of discrete, flexible manufacturing processes.
\end{abstract}

Keywords: Context awareness, Cyber Physical Systems, Flexible Manufacturing System, Process optimization, SOA

\section{Introduction}

Manufacturing industry is nowadays facing several challenges, such as the up-coming customized production, that forces them to move a new concept of FMS. Advanced FMS need to include cyber-physical features, which to assure highest efficiency and availability. This is achvieved by optimization capabilities embedded in the cyber physical features. Such capabilities are needed in wide range of applications: e.g. customized production, maintenance activities, etc.

The work presented in this paper demonstrates how context awareness can be used for optimization of various systems in manufacturing industry, especially flexible manufacturing systems. Key objective thereby is to enable the manufacturing companies to move into a continuous process of increasing efficiency and availability of their production machines [1]. Thereby, the assumption is that cyber-physical features and ICT based context awareness services are suitable technologies that allow for solving the above mentioned problems.

For realizing an ICT solution for run-time optimization of FMS several prerequisites for a context aware infrastructure have to be fulfilled. First, the solutions 
need to be capable of handling high amount of data. Secondly it needs to be capable of handling complex models and algorithms.. Thereby, the key challenge is to find a common approach for addressing the following problems: a) Information on changes need to be gathered in run-time and used for (self) optimization control processes. b) Advanced data processing is needed to allow for enhancement of the gathered information in order to facilitate generation of knowledge about the changes relevant for operation of manufacturing systems.

There is a need for a solution which can be easily applied to various processes and for optimization of different parameters in the processes, instead to build scattered solutions for each process and parameter. This raises the following research question:

What could be a suitable set of methods and tools to allow the realization of reliable adaptable production systems for dynamic manufacturing processes that can be adapted during run-time assuring high availability of such adaptable production systems?

The above stated research question can be addressed by the following hypothesis:

Reliable and highly available production systems for dynamic manufacturing processes can be achieved if a context aware approach is used to identify the current context of manufacturing processes as a basis for adaptation of process parameters and for sharing of knowledge on manufacturing processes.

\section{Context Awareness and Cyber-Physical Systems}

In this paper it is investigated how context awareness can be used to achieve a solution for run-time optimization of various FMS, optimizing high variety of parameters. The context awareness approach allows for observation of changes under which a FMS is operated. The identified context in turn allows for a dynamic adaptation of the FMS to these varying conditions.

Cyber Physical Systems (CPS), integrated in the manufacturing processes offer new opportunities to provide information, which are needed for an effective identification of dynamically changing context under which the observed manufacturing system is operating, during run-time. The assumption is that building and adjustments of such generic context aware solution for various specific optimizations and processes is much more time/costs effective than building of classical optimizations solutions. In order to investigate applicability of a new generic context aware solution to wide scope of complex FMS, in this paper the applications for the two different optimization of two processes is experimentally investigated: process energy consumption optimization (as typical example of so-called secondary manufacturing processes) and process control optimization aiming at higher process efficiency and availability (prime processes).

CPS based on a service oriented architecture (SOA) approach offers completely new possibilities for self-adaptive and context aware solutions. Therefore, it is likely that CPS and SOA based approaches are the most appropriate for the realization of self-adaptive and context aware solutions. By making use of various information sources (sensors, controllers, etc.) through embedded ICT services, such an approach is promising specifically for FMS. 


\section{Related Work}

Context Awareness and Context modelling: Context Awareness is a concept propagated in the domains of ambient intelligence and ubiquitous computing. Existing research on context can be classified in two categories: context-based, proactive delivery of knowledge, and the capture \& utilization of contextual knowledge. In the case of embedded services, the notion of context refers to process preferences of product and process skills of devices, physical capabilities of the equipment and environment conditions. The idea is that computers can be both sensitive and reactive, based on their environment. Using context information is an active area of research, with various context capture methods and context languages defined. Allthough research on context already started back in 1999 [2], [3] and [4], the current research on context is primarily oriented towards capturing and utilization of contextual data for actionable knowledge [5]. Furthermore research shows, that knowledge context could be used to classify and organize knowledge in a networked enterprise [6]. However, provided in [7] shows a lack in provision of knowledge context. Several systems to handle context were proposed by the research community [8] - [12].

By context modeling, the problem of how to represent the context information can be solved. However, how to extract context from the knowledge process and how to manipulate the information to meet the requirement of knowledge enrichment remains to be solved. In the research presented in this paper it is planned to model context with ontologies, and, therefore, context extraction mainly is an issue of context reasoning and context provisioning: how to infer high-level context information from low-level raw context data [13] or monitored sensorial data. Based on the formal description of context information, context can be processed with contextual reasoning mechanisms [14], [15]. The modelling of context in this case presents an additional challenge, especially in highly dynamic and distributed environments [16].

Cyber Physical Systems: In [17] cyber-physical systems (CPS) are defined as '[...] integrations of computation and physical processes [...]'. Research on CPS can be understood as a compound of various disciplines, such as computer science, software engineering systems with mechanical and electrical embedded systems. A CPS represents the idea of combining real-world objects and processes with information processing objects and processes linked together through open, partly global and anytime interconnected information networks like the internet or networking with each other. One example of a typical CPS is an intelligent manufacturing line, where the work of a machine is supported by the communication with its depending components.

Through the coupling of virtual and physical systems, several advantages against conventional computational or embedded systems arise. All accessible data, information and services can be deployed and utilized at any time anywhere in the system. In this way, a series of novel functionalities, properties and services become possible. Thus, cyber-physical systems' services are independent from location, adapted to current systems requirements, partly autonomously, multifunctional and multimodal, networked and distributed along their application area [18], [19]. Therefore, it is expected that CPS will play an important role in future systems, especially also in manufacturing systems [20]. 


\section{Concept for Context Awareness}

The proposed approach to achieve context awareness is summarized in Fig. 1. The objective of the Context Monitoring/Extraction services is to use monitored "raw data" provided by sensors and CPS at the FMS to derive the FMS current contextual situation. Based on the identified current contextual situation, knowledge can be generated that is necessary to provide the basis for operational decisions. This generated knowledge in turn forms the basis for decisions about optimizations of specific manufacturing processes. Decisions regarding the optimization of manufacturing process can be a) short-term (specific taks of a manufacturing process) and b) long-term (overall manufacturing process). The proposed approach provides context awareness about situations and based on this provides an appropriate decision support.

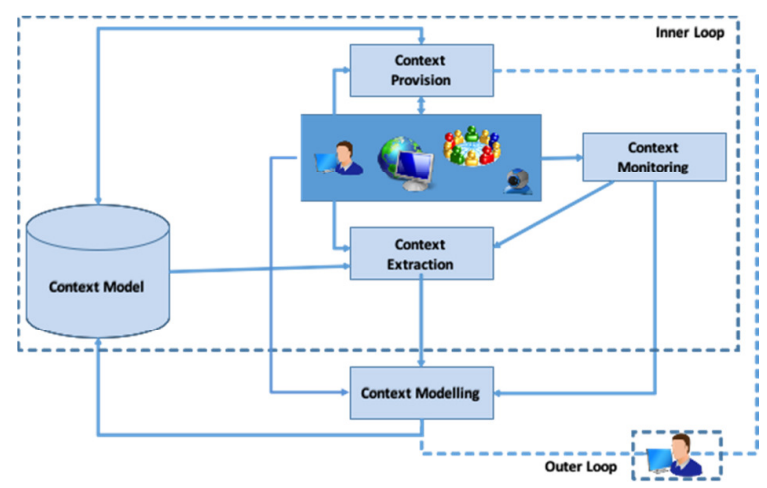

Fig. 1: Context Awareness Concept

Using CPS and other information sources, data on context of manufacturing processes are collected. This information is used to identify the current context of the processes (current approaches often only use location/user for identifying context). The identification is starts through context monitoring services, which are, e.g. services for monitoring of processes or of a user interacting with a system for chaning conditions. The monitored "raw data" is transformed into an "standardized" data format by the monitoring services in order to allow further processing by the context extraction services. The context extraction services identifies current context by instantiating monitored data into the context model. Furthermore, reasoning techniques are used to support context identification. For reasoning previously identified context and the context model is used, which is stored in the context repository. After the current context is identified, it is send to the system adapter services, which are responsible for the system adaptation. In addition, the outer loop supports updating the context. Currently the update is foreseen as manual task, but in future this might be realized as an automatic task.

Context Model: The Context Model forms the foundation for the context extraction. The approach selected to model context is ontology based. Ontologies provide flexibility, expressiveness and extensibility and therefore can be considered as a suitable candidate for representing the context model. They ensure that different 
entities that use the context data have a common semantic understanding of that data. Furthermore, the possibility to easily use reasoning techniques is an advantage of using ontologies. By using such reasoning techniques it is possible to identify inferred knowledge out of the implicitly stated situations. In order to be able to use a context model for context exctraction it is necessary to first define a 'holistic' and dynamic context model. Thereby, it is important to take into account the context of FMS, machines and processes for which context shall be extracted. The ontology based context modelling is promising to be applicable to wide scope of FMS, asking for minimal adjustments developments.

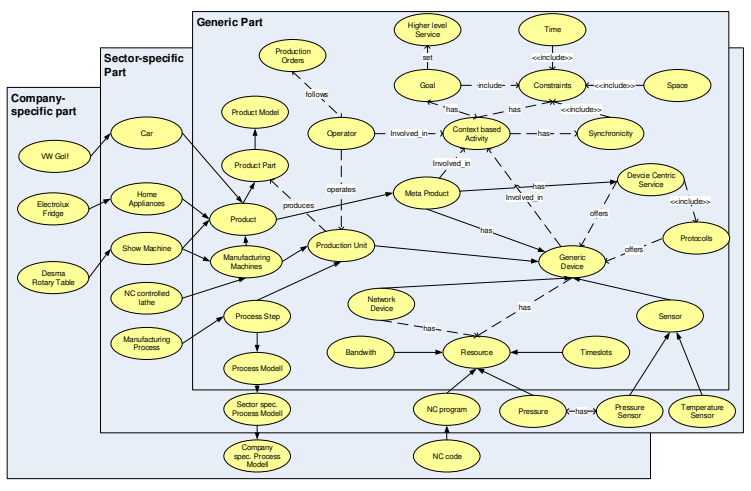

Fig. 2: Proposed Context Model

The context model defines a layered ontology: Generic Context Model, domainspecific model and an application-specific model. The context model includes identification of the set of features that determine the context and, consequently, identification of the set of parameters to be monitored depending on the services to be made. The Context Model is contrary to many current approaches not intended to model "everything", but only the necessary information required for context extraction. Fig. 2 shows an excerpt of an example of the above mentioned layered ontology approach.

\section{Implementation}

A generic solution for a context aware solution is proposed. The proposed solution can be applied to various FMS as an add-on to the existing system. In order to create a common architecture, several application cases from different industrial sectors have been analyzed. Furthermore, results from former research projects (e.g. Self-Learning, U-Qasar, ProSEco) have been re-used. The proposed architecture is shown in Fig. 3 and follows SOA principles. The architecture includes, among others, the following key components:

- The System Monitor receives raw sensor data and provides aggregated data. To achieve this, it allows monitoring of legacy systems in enterprises via different 
interfaces. It is therefore able to correlate data from distinct systems, which later serves as a basis for extraction of contexts.

- The Context Extractor uses the monitored "raw data" provided by the System Monitor to identify the current context of the monitored machine/process. The context is extracted by using the monitored data instantiated in the context model.

- The Context Sensitive System Adapter uses the identified context to update the system behavior. Each adaptation is based on the context knowledge, which defines the rules / parameters to be updated foreach identified context.

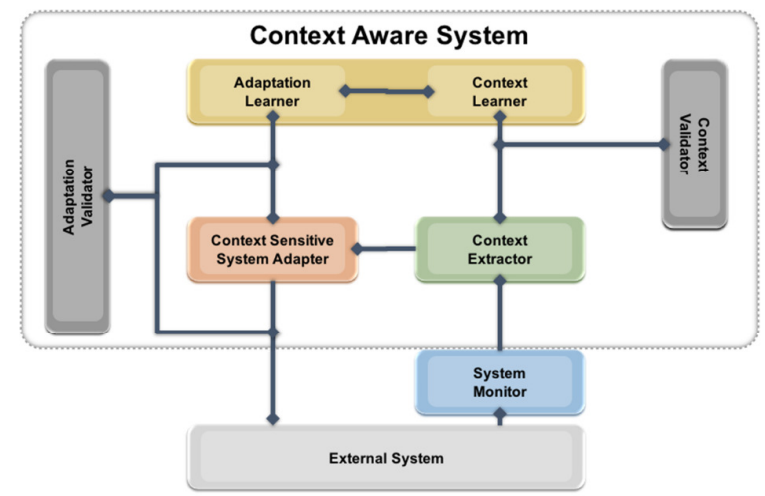

Fig. 3: Proposed Architecture for a Context-Aware System

\section{Early Results}

This experiment involved control systems, machines and automation systems for shoe industry. The production and manufacturing of shoes, especially the production of shoe soles involves various different materials and a large number of automatic as well as manual operations. Such FMS comprise a set of complex operations that depended on the human operator and therefore depend on the operators skills. The need for automatic recognition of current situations and continuous optimisation of processes has been identified by the machine vendors for the shoe industry.

The prposed solution has been integrated into a real installation of a machine vendor for the shoe industry. The company is designing and delivering complex automated machines/systems for the shoe production worldwide. These automation systems, implying rotary table and injection machines, robots etc. The solution is used to identify the operative context within the production process. Based on the identified current context the solution reacts to changing situations associated with variations in different parameter sets in order to improve error-prone processes and reduce maintenance problems. The considered experiment addresses an improved synchronization of valves of an injection machine. The "mixing head" of the injection machine is injecting different materials into a mould through different nonmechanical connected valves. However, after a number of production cycles the valves might get asynchronous due to a variety of influences such as inconstant air 
pressure and/or valve abrasion. Due to asynchronous operation of the valves the quality of the final product is affected negatively. By applying the proposed context aware solution in the injection machine, the valves opening times are automatically adjusted during run-time in order to assure initially planned operation. The proposed solution continuously monitors the process parameters and tries to identify changes in this operative context. Whenever such a change is identified by the proposed solution, the identified context is sent to the system adapter. The system adapter in turn starts an adaptation process, which leads to a set of parameters, that need to be updated in order to adjust the opening times of the valves.

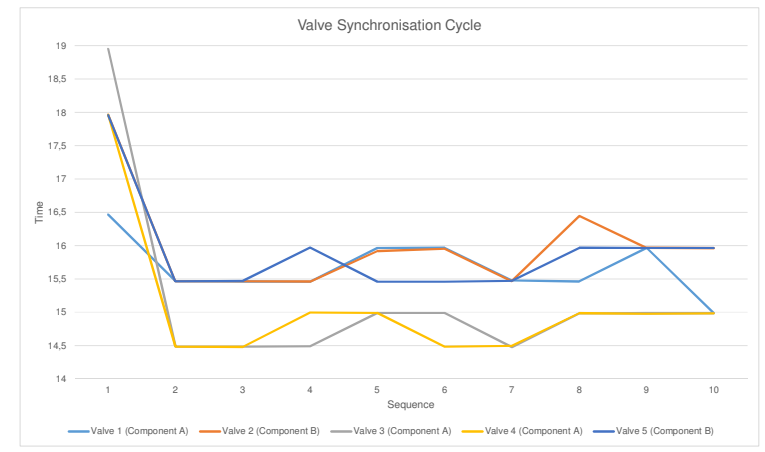

Fig. 4: Results of a Valve Synchronisation test run (including adjustment times)

The results of automatic valve synchronisation are shown in Fig. 4. It shows that the opening times of five valves that are injecting two types of materials (three valves for material A, two valves for material B) are continuously adapted to assure an optimum working range, which is achieved after an initial training phase.

\section{Conclusions and Future Work}

Prototypes of the proposed solution have been implemented and are integrated in real industrial test environment. Currently the approach is running through the testing and evaluation phase. The approach is evaluated in two scenarios and several test cases in different business domains in order to validate the results under different conditions. The innovation of the proposed research are: a) methods on how to achieve context awareness for FMS (this includes guidelines for modelling context) and b) solution to enable the use of context awareness in FMS, e.g. to enable self-adaptation of FMS (this includes services for monitoring and extracting context).

The results of the first executed results in real industrial environments allow for an optimistic expectation regarding future system applicability.

Future research is planned to focus on using additional reasoning capabilities for improving context extraction results. A second topic for further research will be put on methods on how to update the context model(s). Especially for dynamically changing production environments updates of the context model are important. In optimal case these updates should be executed (semi-) automatically. Focus is hereby 
on solving the problem of how to feed updates of the specific context models (domain and application specific) back to the generic context model.

\section{References}

1. Scholze, S., Barata, J., \& Kotte, O. (2013). Context Awareness for self-adaptive and highly available Production Systems. In Technological Innovation for the Internet of. Things, Springer Berlin Heidelberg.

2. Weiser, M., Gold, R. and Brown, J.S., The origins of ubiquitous computing research at PARC in the late 1980s. IBM Systems Journal 38(4), 1999, pp. 693-696

3. Bouquet, P., Giunchiglia, F., van Harmelen, F., Serafini, L., Stuckenschmidt, H., C-OWL: Contextualizing Ontologies, Lecture Notes in Computer Science Volume 2870, 2003.

4. Voida, Stephen and Mynatt, Elizabeth D. and MacIntyre, Blair and Corso, Gregory M., Integrating Virtual and Physical Context to Support Knowledge Workers, IEEE Pervasive Computing archive, Volume 1 Issue 3, July 2002, pp.73-79

5. Mladenić, D., Active Project Website., Retrieved January 10th, 2012, from https://tinyurl.com/jkc23mn

6. Tao, Y., Tianyuan, X., Linxuan, Z., Context-centered design knowledge management, Computer Integrated Manufacturing Systems, 10 (2004), 1541-1545.

7. Ahn, H. J., Lee, H. J., Cho, K., \& Park, S. J., Utilizing knowledge context in virtual collaborative work, Decision Support Systems, 39(2005), 563-582.

8. Bellavista, P., Corradi, A., Montanari, R., Toninelli, A., Context-aware semantic discovery for next generation mobile systems, IEEE Communications Magazine, 44 (2006), 62-71.

9. Toninelli, A., Corradi, A., Montanari, A., Semantic-based discovery to support mobile context-aware service access, Computer Communications, 31 (2008), 935-949.

10. Gu, T., Pung, H. K., Zhang, D. Q., A service-oriented middleware for building contextaware services, Journal of Network and Computer Applications, 28(2005), 1-18

11. Kim, S., Suh, E., Yoo, K., A study of context inference for Web based information systems, Electronic Commerce Research and Applications 6(2007), 146-158.

12. Chang, J. W., Kim, Y. W., 2006, Design and implementation of middleware and context server for context awareness, High Performance Computing and Communications.

13. Scholze, S., Stokic, D., Barata, J., Decker, C., Context Extraction for Self-Learning Production Systems, INDIN 2012, Beijing, 2012

14. Luther, M., et al., Situational reasoning - a practical OWL use case. Chengdu, China, 2005.

15. Forstadius, J., Lassila, O., Seppänen, T., RDF-Based Model for Context-Aware Reasoning in Rich Service Environment, Proceedings of the Third IEEE International Conference on Pervasive Computing and Communications Workshops. IEEE Computer Society, (2005)

16. C. Bettini, O. Brdiczka, K. Henricksen, J. Indulska, D. Nicklas, A. Ranganathan, and D. Riboni, "A survey of context modelling and reasoning techniques," Pervasive Mob. Comput., vol. 6, no. 2, pp. 161-180, Apr. 2010.

17. Lee, E. A., Cyber Physical Systems: Design Challenges, Berkeley UC, 2008.

18. Geisberger, E. and M. Broy, "agendaCPS: Integrierte Forschungsagenda Cyber Physical Systems, National Academy of Science and Engineering," 2012.

19. Bettenhausen, K. D. and S. Kowalewski, Cyber Physical Systems: Chancen und Nutzen aus Sicht der Automation in: Thesen und Handlungsfelder, 2013.

20. Khaitan, S. and J. McCalley, „Design Techniques and Applications of Cyberphysical Systems: A Survey“, Systems Journal, Bd. 9, Nr. 2, pp. 350 - 365, 22 May 2015. 